\title{
How canola and sunflower oils affect lipid profile and anthropometric parameters of participants with dyslipidemia
}

\author{
Sedigheh Saedi ${ }^{1}$, Mona Noroozi ${ }^{1}$, Naghmeh Khosrotabar ${ }^{1}$, Shadi Mazandarani ${ }^{1}$, Behshid Ghadrdoost ${ }^{* 2}$
}

Received: 9 Apr 2016

Published: 15 Jan 2017

\begin{abstract}
Background: Restricted intakes of saturated and trans-fatty acids and replacement with poly or monounsaturated fatty acids are emphasized in healthy diets. This study evaluates the effects of a six-month consumption of canola oil compared to sunflower oil on lipid profile and anthropometric parameters of people affected by dyslipidemia.

Methods: This randomized controlled trial was conducted on 96 patients with dyslipidemia, who were randomly assigned into canola oil or the sunflower oil groups. The participants were instructed to record the contents of their daily meals, beverages, fruits, and snacks a day before treatment, at the second clinic visit, in the third month, and at the end of study (i.e., six months). Lipid profile and anthropometric parameters were compared between the two groups. Student t-test or Mann Whitney U test was used for statistical comparisons of variables between groups. Multivariate analysis was performed to adjust the confounding factor effects.

Results: Of the enrolled participants, $44(45.8 \%)$ were on sunflower oil diet and $52(54.2 \%)$ on canola oil diet. We observed no change in anthropometric parameters and thus no significant difference between the two groups $(\mathrm{p}>0.05)$. Significant reductions in LDL-C $(p<0.001)$, total cholesterol $(p<0.001)$ and triglyceride levels $(p<0.001)$, and significant elevation in HDL-C $(p=0.008)$ were observed in canola oil group, as well as those who used sunflower oil.

Conclusion: Dietary fats in the form of canola oil or sunflower oil effectively lower the serum cholesterol, LDL-C and triglyceride concentrations. They also result in an increase in serum concentration of HDL-C. These oils, however, did not modify general anthropometric parameters.
\end{abstract}

Keywords: Canola Oil, Sunflower Oil, Lipid Profile, Dyslipidemia, Anthropometric Parameters

Copyright $@$ Iran University of Medical Sciences

Cite this article as: Saedi S, Noroozi M, Khosrotabar N, Mazandarani Sh, Ghadrdoost B. Comparison of the effects of canola and sunflower oils on lipid profile and anthropometric parameters of participants with dyslipidemia. Med J Islam Repub Iran. 2017(15 Jan);31.5.

https://doi.org/10.18869/mjiri.31.5

\section{Introduction}

Dyslipidemias are the most common form of metabolic diseases that may lead to atherosclerosis (1). Endothelial cells are continuously exposed to plasma lipoproteins, which affect endothelial function. High levels of total blood cholesterol, low density lipoproteins (LDLs) and fasting triglyceride-rich plasma particles are atherogenic factors that cause endothelium-dependent responses, whereas high density lipoproteins (HDLs) as an antiatherogenic lipoprotein modulate endothelial function in a ben-

Corresponding author: Dr Behshid Ghadrdoost, behshid.ghadrdoost@yahoo.com

1. Rajaie Cardiovascular Medical and Research Center, Iran University of Medical Sciences, Tehran, Iran

2. Physiology Research Center, Iran University of Medical Sciences, Tehran, Iran. eficial manner (2).

There is increasing evidence on the association of fasting plasma LDL and HDL with atherosclerosis. Postprandial hypertriglyceridemia also increases inflammatory agents, such as TNF, and IL-6. Increases in post-prandial cholesterol-rich chylomicron remnants change endothelium integrity by inducing smooth muscle cells and macrophage foam formation, and to which inflammatory cytokines, macrophages and other lipoproteins are added, and

$\uparrow$ What is "already known" in this topic:

Different types of dietary lipids affect serum lipid profile and lipid metabolism in the body. Vegetable oils are known for their cholesterol lowering effects but they differ in their cholesterol lowering capacity.

$\rightarrow$ What this article adds:

Comparing the effects of canola oil and sunflower oil use on lipid profile of healthy volunteers showed that anthropometric parameters remained unchanged during the six months in the participants on either diet. 
affect the shape and function. Different types of dietary lipids affect serum lipid profile and lipid metabolism. Vegetable oils are known for their cholesterol lowering effects when substituted for the dietary animal fat; however, specific types of vegetable oils differ in their cholesterol lowering capacity (3).

Canola oil is a well-known vegetable oil, due to its overall fatty acid (FA) profile low content of saturated fatty acids (SFA) and a high content of polyunsaturated fatty acids (PUFA), including a-linolenic acid (ALA) is favorable in many countries (4).

Another important edible oil is sunflower oil which contains high amount of linoleic acid (PUFA). It is a mixture of oleic acid and stearic acid as major monounsaturated fatty acids (MUFA) and SFA, respectively (5).

Some epidemiological data and randomized controlled trials revealed that replacing saturated fats with PUFA lowered the risk of heart disease events by $24 \%$, and higher omega- 6 diets lowered the risk of coronary heart disease (6). Dietary PUFAs, such as canola oil or sunflower oil affect a wide variety of physiological processes, but investigators in nutrition science are now trying to clarify the pathways and mechanisms for the biological action of n-3 PUFAs (7).

Since dietary PUFAs of the n-6 and n-3 series and changes in the lipid profile have a role in cardiovascular events, in this study we decided to evaluate the effects of two commonly used forms of dietary oils (canola and sunflower) in the general population on the lipid profile of healthy volunteers in a randomized controlled trial.

\section{Methods}

Study Design

This randomized controlled trial commenced with 105 patients who had dyslipidemia as defined by higher proportions of LDL-C, reduced HDL-C, and increased triglycerides (8). These participants referred to the lipid clinic at Rajaie Cardiovascular Medical and Research Center to check their blood lipid profile. None of the patients was using lipid-lowering drugs. The participants had no history of renal or liver disease. Those with a history of diabetes mellitus, thyroid dysfunction or alcohol consumption and supplement intake were excluded. Most of the participants had low (level 1) daily physical activity.

Among the 105 participants who participated in the study, nine decided to leave the study, because they were unable to comply with the consumption of a specified meal for more than three sequential days. Therefore, the data of these participants were excluded. Consequently, the study was conducted on 96 participants with the mean age of $51.39 \pm 12.85$, and the mean body mass index (BMI) of $27.71 \pm 4.01 \mathrm{~kg} / \mathrm{m} 2$.

The study protocol was approved in Ethis Committee of Iran University of Medical Sciences and informed consent were taken from all participants. They were also informed about the study aims and expected results (2000).

\section{Randomization}

Eligible participants were assessed in terms of body weight, height and anthropometric measurements at the first visit in the clinic after screening. The procedures were explained to the participants in detail and they all

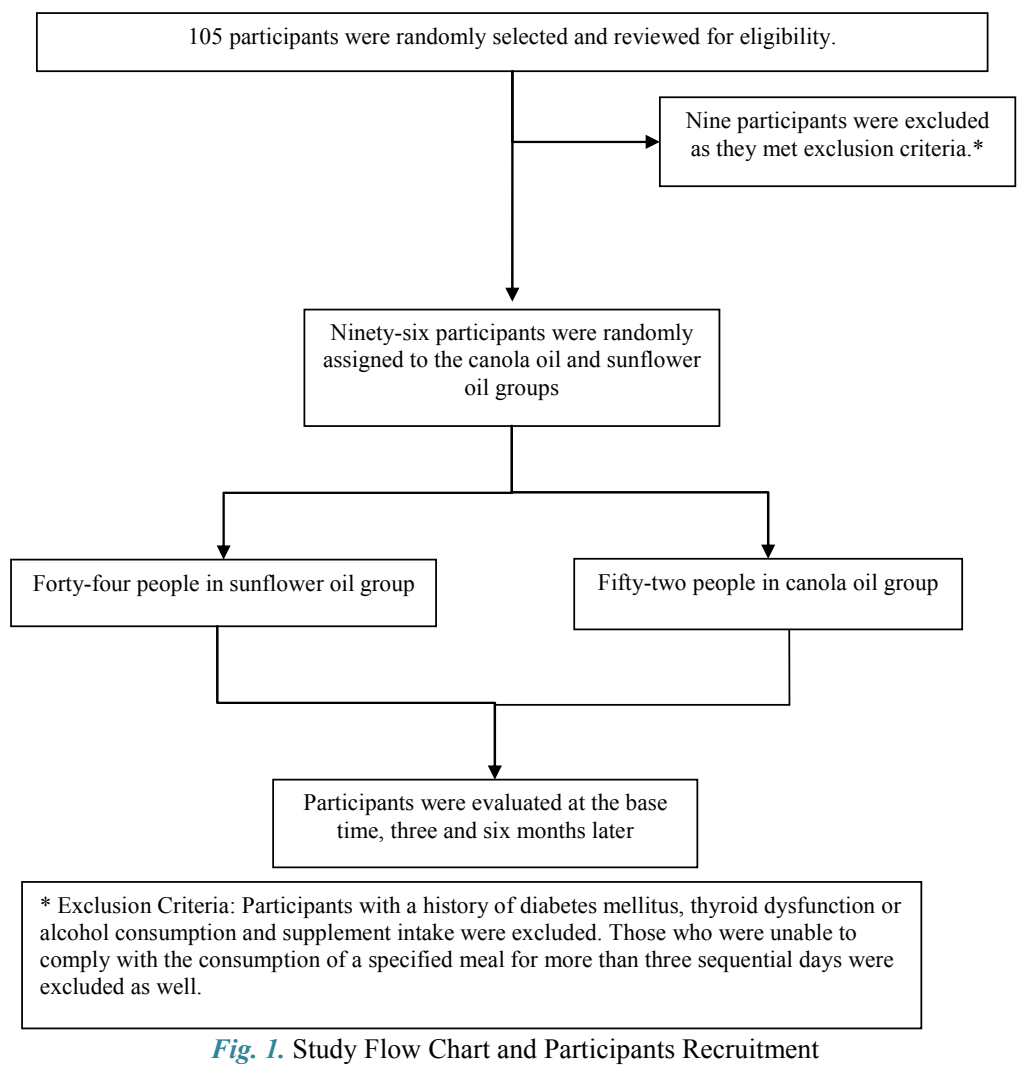


provided us with signed informed consent before the study. Then they were randomly assigned to canola oil or the sunflower oil groups, and study products were dispensed to them. Consort flow diagram of the study is depicted in Figure 1.

All patients were advised not to change their diet and level of physical activity during the study. Nina Company (FRICO, Tehran, Iran) provided both types of oils used in this study. Participants were asked to refrain from the use of canola and sunflower oil for two weeks prior to the study, and only use corn oil for cooking. In addition, they were asked to replace their usual edible oil with canola oil or sunflower oil during the six-month period of the study and do not use other types of oils.

The participants were instructed to record the contents of their daily meals, beverages, fruits, and snacks in a diet at a day before treatment, at the second visit in the clinic, in the third month, and at the end of the study, and after six months. Daily intakes of energy, fat, protein and carbohydrate were calculated and analyzed from the dietary records by Nut4 software.

\section{Anthropometric Measurements}

Body weight, and waist and hip circumference were measured at baseline, in the middle (three-month) and at end of the study. Height was measured prior to the treatment, in centimeters, without shoes. Weight was measured in kilograms without shoes and with minimal clothing by analog scales Seca with an accuracy of 1000 gram.

\section{Laboratory Measurements}

Lipid profiles including total cholesterol (TC), LDLcholesterol (LDL), HDL-cholesterol (HDL), and triglycerides (TG) were measured twice on separate days at baseline and at the end of each six- month treatment period. Blood samples were collected from the participants at 9:00 o'clock, following an overnight fast starting 21:00 o'clock on the previous day. Enzyme assay kits were used, Audit/ Ireland.

\section{Statistical Analysis}

Statistical analysis was performed with SPSS 15. Continuous data were presented as mean $\pm \mathrm{SD}$, and categorical data were expressed as number (\%). All variables were tested for normal distribution, using KolmogorovSmirnov test. Student t-test or Mann Whitney U test was used for statistical comparisons of variables between groups. Multivariate analysis was performed to adjust the confounding factor effects. $\mathrm{P}<0.05$ were considered significant.

\section{Results}

A total of 96 patients with dyslipidemia were enrolled. Of the enrolled participants, $44(45.8 \%)$ were on sunflower oil diet and $52(54.2 \%)$ on canola oil diet. We were able to achieve complete follow up in all 96 patients. The baseline characteristics of the participants of the two groups are demonstrated in Table 1. We found no major differences between the two groups with respect to baseline characteristics.

Level of physical activity is depicted in Table 2. There were no significant differences between groups based on physical activity $(p=0.90)$. Most of the participants had low activity levels (44.2\% in canola group vs. $43.2 \%$ in sunflower group).

\section{Energy Consumption}

Before the treatment, there were no statistically significant differences between the canola oil group and the sunflower oil group with respect to energy, fat or protein intake, but carbohydrate intake was higher in the sunflower group than the canola group $(\mathrm{p}=0.04)$ (Table 3$)$.

No significant differences were detected between the two groups in energy intake and major nutrient intake during the study and at the end of the study. We found that diet condition remained generally constant and comparable during the study period.

Table1. Baseline characteristics in participants on canola oil diet and corn oil diet

\begin{tabular}{llll}
\hline & Canola Oil $(\mathrm{n}=52)$ & Sunflower Oil $(\mathrm{n}=44)$ & $\mathrm{p}$ \\
\hline Age & $51.94 \pm 12.96$ & $50.75 \pm 12.83$ & 0.53 \\
BMI & $28.02 \pm 4.17$ & $27.34 \pm 3.82$ & 0.41 \\
Sex (M/F) & $27 / 25$ & $20 / 22$ & 0.72 \\
Risk Factors & & & \\
HTN & $9(17.3 \%)$ & $4(9.1 \%)$ & 0.24 \\
Smoking & $4(7.7 \%)$ & $7(15.9 \%)$ & 0.20 \\
History of Cardiac Diseases & $4(7.7 \%)$ & $5(11.6 \%)$ & 0.51 \\
Drugs & & & 0.74 \\
Statins & $17(32.7 \%)$ & $13(29.5 \%)$ & 0.49 \\
Beta blockers & $10(19.2 \%)$ & $11(25 \%)$ & \\
\hline BMI: Body Mass Index, HTN:Hyperten
\end{tabular}

BMI: Body Mass Index, HTN: Hypertension

Statistical Methods: Chi-square, student t-test

Table 2. Level of physical activity in canola and sunflower oil groups

\begin{tabular}{llll}
\hline & Canola Oil & Sunflower oil & $\mathrm{p}$ \\
\hline Low & $43(73 \%)$ & $33(72.7 \%)$ & 0.90 \\
Active & $13(25 \%)$ & $10(22.7 \%)$ & \\
Very active & $1(1.9 \%)$ & $2(4.5 \%)$ & \\
\hline
\end{tabular}

Statistical Methods: Mann-Whiney U test 
Table 3. Intakes of Energy and Major Nutrients Consuming either Canola Oil or Sunflower Oil Diet during Six Months

\begin{tabular}{|c|c|c|c|c|}
\hline & Time & Canola Oil & Sunflower Oil & $\mathrm{p}$ \\
\hline \multirow[t]{3}{*}{ Energy (kcal/day) } & Baseline & $1700.6 \pm 489.2$ & $1603.5 \pm 397.9$ & 0.29 \\
\hline & Third month & $1716.3 \pm 370.1$ & $1759.1 \pm 311.5$ & 0.53 \\
\hline & Sixth month & $1684.3 \pm 339.0$ & $1661.3 \pm 281.7$ & 0.68 \\
\hline \multirow[t]{3}{*}{ Fat (g/day) } & Baseline & $53.66 \pm 17.31$ & $51.88 \pm 15.03$ & 0.50 \\
\hline & Third month & $54.87 \pm 12.79$ & $59.90 \pm 15.72$ & 0.09 \\
\hline & Sixth month & $53.93 \pm 12.92$ & $55.79 \pm 15.40$ & 0.57 \\
\hline \multirow[t]{3}{*}{ Protein (g/day) } & Baseline & $87.82 \pm 30.13$ & $91.79 \pm 19.63$ & 0.81 \\
\hline & Third month & $91.79 \pm 19.63$ & $96.96 \pm 18.47$ & 0.19 \\
\hline & Sixth month & $92.05 \pm 26.00$ & $90.75 \pm 18.49$ & 0.68 \\
\hline \multirow[t]{3}{*}{ Carbohydrate (g/day) } & Baseline & $214.46 \pm 75.49$ & $186.95 \pm 62.67$ & 0.04 \\
\hline & Third month & $202.43 \pm 57.82$ & $199.93 \pm 48.17$ & 0.82 \\
\hline & Sixth month & $201.11 \pm 57.34$ & $188.32 \pm 41.79$ & 0.42 \\
\hline \multicolumn{5}{|c|}{ Statistical Methods: Mann-Whiney U test or T Student-test } \\
\hline & & Canola Oil & Sunflower Oil & $\mathrm{p}$ \\
\hline \multirow[t]{3}{*}{ Weight } & & $78.68 \pm 14.89$ & $75.62 \pm 13.07$ & 0.28 \\
\hline & onth & $78.75 \pm 14.40$ & $76.23 \pm 13.27$ & 0.27 \\
\hline & onth & $78.25 \pm 14.36$ & $76.04 \pm 13.19$ & 0.43 \\
\hline \multirow[t]{3}{*}{ Waist } & & $95.90 \pm 14.04$ & $95.63 \pm 12.46$ & 0.91 \\
\hline & onth & $96.01 \pm 13.88$ & $96.18 \pm 12.27$ & 0.96 \\
\hline & onth & $95.53 \pm 13.89$ & $96.00 \pm 12.31$ & 0.86 \\
\hline \multirow[t]{3}{*}{ Hip } & & $106.98 \pm 10.39$ & $106.18 \pm 9.71$ & 0.85 \\
\hline & onth & $107.00 \pm 10.25$ & $106.72 \pm 9.78$ & 0.95 \\
\hline & onth & $106.69 \pm 10.24$ & $106.45 \pm 6.62$ & 0.78 \\
\hline \multirow[t]{3}{*}{ BMI } & & $28.02 \pm 4.17$ & $27.34 \pm 3.82$ & 0.41 \\
\hline & onth & $28.05 \pm 4.04$ & $27.55 \pm 3.84$ & 0.53 \\
\hline & onth & $27.88 \pm 4.11$ & $27.48 \pm 3.80$ & 0.62 \\
\hline
\end{tabular}

BMI: Body Mass Index

Statistical Methods: Mann-Whiney U test or T Student-test

Table 5. Comparison of Lipid Profile in Canola Oil Group and Sunflower Oil Group

\begin{tabular}{|c|c|c|c|c|}
\hline & & Canola Oil & Sunflower Oil & $\mathrm{p}$ \\
\hline \multirow[t]{3}{*}{$\mathrm{TC}$} & Baseline & $220.13 \pm 41.94$ & $223.95 \pm 43.81$ & 0.82 \\
\hline & Sixth month & $194.31 \pm 39.45$ & $196.45 \pm 35.28$ & 0.75 \\
\hline & & $<0.001$ & $<0.001$ & \\
\hline \multirow[t]{3}{*}{ LDL-C } & Baseline & $129.54 \pm 39.74$ & $140.55 \pm 41.29$ & 0.22 \\
\hline & Sixth month & $116.12 \pm 35.83$ & $122.95 \pm 32.97$ & 0.37 \\
\hline & & $<0.001$ & $<0.001$ & \\
\hline \multirow[t]{3}{*}{ HDL-C } & Baseline & $44.24 \pm 12.31$ & $44.82 \pm 12.29$ & 0.62 \\
\hline & Sixth month & $47.28 \pm 11.90$ & $46.73 \pm 11.35$ & 0.56 \\
\hline & & 0.008 & 0.002 & \\
\hline \multirow[t]{3}{*}{ TG } & Baseline & $197.14 \pm 103.23$ & $189.02 \pm 110.02$ & 0.61 \\
\hline & Sixth month & $172.49 \pm 78.25$ & $171.04 \pm 88.69$ & 0.75 \\
\hline & & $<0.001$ & 0.007 & \\
\hline \multirow[t]{3}{*}{ LDL/HDL } & Baseline & $3.02 \pm 1.48$ & $3.22 \pm 0.95$ & 0.16 \\
\hline & Sixth month & $2.48 \pm 0.79$ & $2.96 \pm 0.75$ & 0.18 \\
\hline & & 0.007 & 0.001 & \\
\hline \multirow[t]{3}{*}{ TC/HDL } & Baseline & $5.34 \pm 2.05$ & $5.15 \pm 1.15$ & 0.68 \\
\hline & Sixth month & $4.27 \pm 1.32$ & $4.30 \pm 0.92$ & 0.90 \\
\hline & & $<0.001$ & $<0.001$ & \\
\hline
\end{tabular}

TC: Total cholesterol, LDL-C: Low Density Lipoprotein-Cholesterol, HDL: High Density Lipoprotein-Cholesterol, TG: Triglycerides

Statistical Methods: Mann-Whiney U test or T student-test, Pair T test

\section{Anthropometric Parameters}

Changes in the anthropometric parameters during the study are summarized in Table 4. Body weight and BMI remained constant and no significant difference was observed between the two groups. Waist and hip circumference were maintained during the test period, and no significant differences were noted between the groups.

\section{Blood Chemistry}

Lipid parameters (TG, TC, LDL, HDL) were changed during the study, though the changes were not significant (Table 5).

The serum total cholesterol concentration was signifi- cantly decreased after six months in both groups. In canola oil group, mean \pm SD of TC level was $220.13 \pm 41.94$ at the baseline, which was reduced to $194.31 \pm 39.45$ $(\mathrm{p}<0.001)$; and it was $223.95 \pm 43.81$ at the baseline in the sunflower oil group, which was decreased to $196.45 \pm 35.28(\mathrm{p}<0.001)$. There were no statistically significant differences between the two groups in the total cholesterol prior to the study and after six months.

Although the trend of LDL cholesterol concentration was not statistically different between the two groups, reduction of mean \pm SD of LDL level in each of the groups was significant during the study period. It was $129.54 \pm 39.74$ at the baseline in the canola oil group that 
was changed to $116.12 \pm 35.83(\mathrm{p}<0.001)$, and it was $140.55 \pm 41.29$ for the sunflower oil group that was decreased to $122.95 \pm 32.97(\mathrm{p}<0.001)$.

The changes in serum HDL cholesterol concentration were significant in both groups. HDL level was $44.24 \pm 12.31$ at the baseline that was increased to $47.28 \pm 11.90$ (p-value: 0.008 ) in the canola oil group, and it was $44.82 \pm 12.29$ in the sunflower oil group that was increased to $46.73 \pm 11.35$ ( $\mathrm{p}=0.002$ ). There were no statistically significant differences between the two groups in HDL concentration at the baseline and after six months.

The serum TG concentration significantly decreased in both groups after six months. In the canola oil group, the mean \pm SD of TG level was $197.14 \pm 103.23$ at baseline, which was reduced to $172.49 \pm 78.25(\mathrm{p}<0.001)$, and it was $189.02 \pm 110.02$ at baseline in the sunflower oil group, which was decreased to $171.04 \pm 88.69(\mathrm{p}=0.007)$. Although the decrease in TG level was greater in the canola oil group compared to the sunflower oil group, this reduction was not statistically significant.

No significant differences were found between groups in TC/HDL and LDL/HDL ratio $(p>0.05)$, but reduction of TC/HDL and LDL/HDL was statistically significant in each group (Table 5).

Multivariate analysis, using repeated measure ANOVA, was performed to adjust the effect of baseline carbohydrate. Table 6 demonstrates that different baseline carbohydrates had no effect on the serum lipid profile and on the anthropometric results during the time in the two groups.

\section{Discussion}

The safety of canola oil for intended use in foods has been evaluated and approved by the United States Food and Drug Administration (FDA) (9). Consumption of canola oil is now high in many countries, because it is considered to be a healthy oil and partly because of its low price. Canola oil, also known as high oleic acid content, is low in saturated fat, and contains both omega- 6 and omega-3 fatty acids in ratio $2: 1$, which is supposed to reduce low density lipoprotein and overall cholesterol levels.

Hypocholesterolemic effect of oleic acid is due to a decrease in LDL-C (10). Because of high oleic acid content, canola oil is presumed to be more beneficial than olive and sunflower oils. However, no significant differences were reported between canola oil and the other vegetable oil in HDL-C concentration. Canola oil has neutral effects on TAG levels, omega 3, and high oleic acid content of canola maintains the TAG levels in normal ranges. The effect of canola oil on TAG proves its role in CHD by regulating lipids in blood circulation (11).

Canola oil is also believed to reduce the risk of coronary heart disease and all-cause and cardiovascular mortality due to its unsaturated fat content (4). Sunflower oil contains appreciable quantities of vitamin E, sterols, squalene, and other aliphatic hydrocarbons. The combination of fatty acids in the body is extremely important to maintain various elements of human health, and sunflower oil can help maintain that balance (12).

The aim of this study was to evaluate the effects of can- ola oil, compared to sunflower oil, on anthropometric parameters and blood lipid profile during a six- month period in patients with dyslipidemia. The results revealed that using canola oil in the diet by men and women with dyslipidemia resulted in a significant reduction in LDL-C from baseline $(p<0.001)$, total cholesterol $(p<0.001)$, and triglyceride $(p<0.001)$, and significant elevation in HDL-C from baseline $(\mathrm{p}=0.008)$. However, similar changes were observed in the group who used sunflower oil.

A study by Gulesserian et al. demonstrated that a fivemonth canola oil diet reduced total and LDL cholesterol in children and adolescents with familial hypercholesterolemia, but their HDL-C and LP (a) were unaffected (13). Bierenbaum et al. assessed a $30 \mathrm{ml} /$ day of canola oil in the routine diet of those with dyslipidemia in a cohort study, and concluded that serum LDL decreased significantly, but TC and HDL-C did not change significantly (14). Longer duration of canola oil use by patients in these studies and higher mean of baseline LDL-C, compared to our results $(\mathrm{LDL}=\mathrm{c}$ : $173 \pm 9$ vs. $129 \pm 39$ in our study), may explain the differences in the results.

Other studies showed similar results for TC, LDL-C and TG, which were reduced, but almost equally in both diets; i.e., canola and sunflower oil. Gustafsson et al. studied the effects of these two diets on participants with moderate hyperlipoproteinemia for three weeks, and observed that dietary fat in the form of MUFAs or PUFAs effectively reduced serum total cholesterol, LDL-C and serum TG concentration, but more prominently in sunflower oil group rather than the canola oil group (15). However, Valsta et al. noted a more efficient reduction of LDL-C in canola oil diet than sunflower oil diet (16).

Lambert et al reported a significant reduction in total cholesterol and LDL-cholesterol after a 12-week sunflower oil supplementation period. Although it reduced the HDL cholesterol concentration in women after the 12week sunflower oil supplementation period, it was attributed to weight loss of the participants (17).

A growing body of evidence demonstrates that the consumption of at least 5 to $10 \%$ of energy from omega- 6 or 3 PUFAs reduces the risk of cardiovascular disease and can prevent sudden death in patients with acute coronary syndrome in addition to good lifestyle and dietary regimen (18).

The effects of canola oil consumption on coronary heart disease were evaluated in many studies, and significant reductions were observed in the total cholesterol and LDL- cholesterol, as well as increased tocopherol levels and improved insulin sensitivity $(10,19)$.

On the other hand, sunflower oil has shown few effects on the cardiovascular system, apart from its beneficial effect on the serum lipid profile (5).

Both canola and sunflower oils are high in the essential vitamin E. Vitamin E is well recognized for its antioxidant role and protection from reactive oxygen species, which has been implicated in atherosclerosis (20). There is a high correlation between serum lipids and concentration of Vitamin E (21).

To summarize, this study was conducted to investigate the effects of canola oil diet, compared to sunflower oil 
diet, on anthropometric parameters, including weight, waist circumference and hip circumference and BMI. We found that anthropometric parameters remained unchanged during the six months in the participants on either diet, which seems to be due to replacing comparable amounts of saturated fat in the diet without increasing calorie intake.

\section{Conclusion}

In conclusion, both dietary fats in the form of canola oil or sunflower oil effectively reduce the serum cholesterol, LDL-C concentration and triglyceride concentration. An increase in serum concentrations of HDL-C occurred in both diets. No significant effect was found on anthropometric parameters in this study.

A limitation of this study was that the amount of energy intake, and MUFA and PUFA contents were not carefully measured. In fact, all participants had their regular diet, and the only difference was that oil consumption had been changed.

Another limitation of this study was different levels of dyslipidemia in the study population. Additional research is needed to evaluate the effects of canola oil within a lipid-lowering diet in participants with different stages of dyslipidemias over longer periods.

\section{Acknowledgments}

None.

\section{Conflict of Interest: None declared.}

\section{References}

1. Akram Shad M, Iqbal T, Hussain Shah M. Vegetable oils and dyslipidemia. Pak J Med Sci. 2003;19(1)45-51

2. Sitia S, Tomasoni L, Atzeni F, Ambrosio G, et al. From endothelial dysfunction to atherosclerosis. Autoimmun Rev. 2010 Oct;9(12):8304.

3. Jones PJ, MacKay DS, Senanayake VK, Pu S, Jenkins DJ, Connelly PW, et al. High-oleic canola oil consumption enriches LDL particle cholesteryl oleate content and reduces LDL proteoglycan binding in humans. Atherosclerosis. 2015;238:231e238

4. Hoffman R, Gerber M. Can rapeseed oil replace olive oil as part of a Mediterranean-style diet? Br J Nutri. 2014;112:1882-1895.

5. BesterD, Esterhuyse AJ, Truter EJ, Rooyen JV. Cardiovascular effects of edible oils: a comparison between four popular edible oils. Nutr Res Rev. 2010;23:334-348.

6. Harris WS, Mozaffarian D, Rimm E, Kris-Etherton P, Rudel LL, Appel LJ, et al. Omega 6 fatty acids and risk for cardiovascular disease: sc-ience advisory from the American Heart Association Nutrition Subcommittee of the Council on Nutrition, Physic Activity, and Metabolism; Council on Cardiovascular Nursing; and Council on Epidemiology and Prevention. Circulation. 2009 Feb;119(6): 902-7.

7. Ander BP, Dupasquier CM, Prociuk M, Pierce G. Polyunsatu-rated fatty acids and their effects on cardiovascular disease. Exp Clin Cardiol. $2003 ; 8(4): 164-72$.

8. Musunuru K. Atherogenic Dyslipidemia: Cardiovascular Risk and Dietary Intervention. Lipids. 2010 Oct;45(10):907-914.

9. U.S. Food and Drug Administration. Qualified Health Claims: Letter of Enforcement Discretion - Unsaturated Fatty Acids from Canola Oil and Reduced Risk of Coronary Heart Disease (Docket No. 2006Q0091). Available from: http://www.fda. gov/Food/ IngredientsPackagingLabeling/LabelingNutrition/ucm072958.htm.

10. Lin L, Allemekinders H, Dansby A, Campbell L, Durance-Tod S, Berger A, et al. Evidence of health benefits of canola oil. Nutr Rev. 2013 Jun; 71(6):370-85.

11. KostikV, Memeti Sh, Bauer B. Fatty acid composition of edible oils and fats. J Hyg Engin Design. 2013;4:112-116.
12. Christov M. Contribution of interspecific hybridization to sunflower breeding. HELIA 2012;35 (57):37-46.

13. Gulesserian T, Widhalm K. Effect of a rapeseed oil substituting diet on serum lipids and lipoproteins in children and adolescents with familial hypercholesterolemia. J Am Coll Nutr .2002 Apr; 21(2):103-8.

14. Bierenbaum ML, Reichstein RP, Watkins TR, Maginnis WP, Geller M. Effects of canola oil on serum lipids in humans. J Am Coll Nutr. 1991 Jun; 10(3):228-33.

15. Gustafsson IB, Vessby B, Ohrvall M, Nydahl M. A diet rich in monounsaturated rapeseed oil reduces the lipoprotein cholesterol concentration and increases the relative content of $\mathrm{n}-3$ fatty acids in serum in hyperlipidemic subjects. Am J Clin Nutr. 1994 Mar;59(3):667-74.

16. Valsta LM, Jauhiainen M, Aro A, Katan MB, Mutanen M. Effects of a monounsaturated rapeseed oil and a polyunsaturated sunflower oil diet on lipoprotein levels in humans. Arterioscler Thromb Vasc Boil. 1992;12:50-57.

17. Lambert EV, Goedecke JH, Bluett K, et al. Conjugated linoleic acid versus high-oleic acid sunflower oil: effects on energy metabolism, glucose tolerance, blood lipids, appetite and body composition in regularly exercising individuals. Br J Nutr. 2007;97:1001-1011.

18. Ghanbari B, Khaleghparast S, Ghadrdoost B, Bakhshandeh H. Nutritional status and coronary artery disease: a cross sectional study. Iran Red Crescent Med J. 2014 Mar;16(3):e13841.

19. Gebauer SK, Psota TL, Harris WS, et al. n-3 fatty acid dietary recommendations and food sources to achieve essentiality and cardiovascular benefits. Am J Clin Nutr. 2006;83(6 Suppl):1526S-1535S.

20. Okosun RE, Adu ME. Effect of Oral Vitamin E on Serum Lipid Profile of Apparently Healthy Nigerians in Benin City. Trop J Pharmaceut Res. 2015;14 (6):1071-1074.

Khajehdehi P. Effect of vitamins on lipid profile of patients on regular haemodialysis. Scand J Urol Nephrol. 2000;34:64-66. 